\title{
Acid survival of Escherichia coli; how dead is dead?
}

\author{
V. Wong ${ }^{1}$, J. Liversidge ${ }^{1}$, M. G. Blacklock ${ }^{1}$, L. Duncan ${ }^{1}$, A. J. Harding ${ }^{2}$ and I. R. Booth ${ }^{2}$ \\ ${ }^{1}$ School of Medicine, University of Aberdeen, Aberdeen, UK and ${ }^{2}$ School of Medical Sciences, University of Aberdeen, \\ Aberdeen, $U K$
}

Escherichia coli is a significant food-poisoning pathogen and recent outbreaks have caused increased concerns from experts in the field of nutrition and food science. Population diversity demonstrated by commensal E. coli when growing exponentially in a defined medium is most evident in the formation of tailing populations of surviving organisms when the population is subjected to extreme stress ${ }^{(1)}$. The present study describes the fluorescence-activated cell sorting (FACS)-based analysis of $E$. coli acid survival.

Acid killing was performed on purified commensal E. coli strain J1. Culture of E. coli strain J1 in mid-exponential phase was subjected to extreme $\mathrm{pH}$ stress at $\mathrm{pH} 3$, with $E$. coli strain Frag 1 as control. A viable colony count was performed to estimate the rate at which cells die. Cell viability was assessed using a BacLight kit (Invitrogen Ltd, Paisley, UK). J1 cells were stained using a BacLight kit containing SYTO9 and propidium iodide stains (Invitrogen Ltd). For FACS analysis fully-stained J1 cell populations were prepared and incubated in a medium at either $\mathrm{pH} 7$ or $\mathrm{pH} 3$ for different time intervals. Samples were centrifuged, resuspended in a minimal medium (pH 7) without growth supplements and scanned using an FACS DIVA cell sorter (Becton Dickinson UK Ltd, Oxford, Oxon., UK). Windows were defined based on controls. Once a window had been defined to separate cells from the population based on viable and non-viable properties, cells were allowed to rest anaerobically in the minimal medium. After a $1 \mathrm{~h}$ resting period cells were re-scanned.

Acid-killing experiments revealed that $E$. coli strain $\mathrm{J} 1$ survives extreme $\mathrm{pH}$ stress $(\mathrm{pH} 3$ ) better than strain Frag 1, and a surviving population of J1 was identified. From FACS analysis J1 cells incubated in a medium at pH 7 or pH 3 were highly fluorescent with SYTO9, with cells incubated at $\mathrm{pH} 3$ showing progressive loss of SYTO9 fluorescence with increasing incubation period. When a second scan was conducted at approximately $60 \mathrm{~min}$ after resuspension in the minimal medium at $\mathrm{pH} 7$, cells incubated at $\mathrm{pH} 3$ for 10 min and rested for $60 \mathrm{~min}$ at $\mathrm{pH} 7$ were observed to divide into two populations, one population shifted towards the viable end with higher SYTO9 fluorescence than before, while the other population remained in the same position. Cells incubated at pH 3 for 20 min and rested for $60 \mathrm{~min}$ at $\mathrm{pH} 7$ were also observed to divide into two populations; one population showed a reduced SYTO9 fluorescence and hence shifted towards the non-viable end, while the other population remained in the same position. Cells incubated at $\mathrm{pH} 3$ for $30 \mathrm{~min}$ and $40 \mathrm{~min}$ before being rested for $60 \mathrm{~min}$ at $\mathrm{pH} 7$ showed a shift towards the non-viable end. No propidium iodide fluorescence was observed.

The results suggest that cells do die during incubation in the medium at $\mathrm{pH} 3$; however, some cells are more resistant and may be able to recover and repair DNA with time when $\mathrm{pH}$ is restored. Fewer cells recover with increasing period of incubation at $\mathrm{pH} 3$. As there may be recovering cells that are perceived to be dead in current food diagnostic procedures involving the use of the BacLight kit, these results may help enhance current procedures to ensure that food products are free from contamination.

1. Booth IR (2002) Int J Food Microbiol 78, 19-30. 